\title{
Entropy for Past Residual Life Time Distributions
}

\author{
Arif Habib $^{1}$, Meshiel Alalyani ${ }^{2}$ \\ ${ }^{1}$ College of applied Medical Sciences - Khamis Mushait;,King Khalid University, Kingdome of Saudi Arabia \\ ${ }^{2}$ College of Nursing - Khamis Mushait, King Khalid University, Kingdome of Saudi Arabia
}

\section{Email address:}

ahabib@kku.edu.sa (A. Habib), malalyani@kku.edu.sa (M. Alahyani)

\section{To cite this article:}

Arif Habib, Meshiel Alahyani. Entropy for Past Residual Life Time Distributions. American Journal of Theoretical and Applied Statistics. Vol. 4, No. 3, 2015, pp. 118-124. doi: 10.11648/j.ajtas.20150403.17

\begin{abstract}
As we are familiar that existence of life is uncertain. In the context of reliability and lifetime distributions, there are some measures such as the hazard rate function or the mean residual lifetime function that have been used to characterize or compare the aging process of a component. This definition deals with random variable truncated above some t, i.e. the support of the random variable is taken to be $(0, t)$. We outline some common methods for past residual lifetime distributions with the aim to provide some insights on general construction mechanisms. Some applications are given to provide the readers a possible source of ideas to draw upon. Applications of past residual lifetime distributions in reliability, survival analysis and mortality studies are briefly discussed.
\end{abstract}

Keywords: Differential Entropy, Past Residual Entropy, Life Time Distributions

\section{Introduction}

Information theory includes the study of uncertainty measures plays a significant role in studying the various aspects of a system when it fails between two time points. In reliability theory and survival analysis, the residual entropy was considered in Ebrahimi and Pellerey [2], which basically measures the expected uncertainty contained in remaining lifetime of a system. The residual entropy has been used to measure the wear and tear of components and to characterize, classify and order distributions of lifetimes by Belzunce et al. [3] and Ebrahimi [4]. The notion of past entropy, which can be viewed as the entropy of the inactivity time of a system, was introduced in Di Crescenzo and Longobardi [5]. Many ageing (lifetime) distributions have been constructed with a view for applications in various disciplines, in particular, in reliability engineering, survival analysis, demography, actuarial study and others. Statistical analysis of lifetime data is an important topic in biomedical science, reliability engineering, social sciences and others. Typically, 'lifetime' refers to human life length, the life span of a device before it fails, the survival time of a patient with serious disease from the date of diagnosis or major treatment or the duration of a social event such as marriage.

Let $X$ be an absolutely continuous non- negative random variable having distribution function $F(x)$ and survival function $R(x)$. The basic measure of uncertainty is defined by Shannon [22] as

$$
H(X)=-\int_{0}^{\infty} f(x) \log f(x) d x
$$

where $f(x)$ is the density function of $X$. (Throughout this paper, $\log$ will denote the natural logarithm.)

If $X$ is a discrete random variable taking the values $x_{1}, x_{2}, \ldots, x_{n}$ with respective probabilities $p_{1}, p_{2}, \ldots, p_{n}$, then the Shannon's entropy is defined as

$$
H(P)=H\left(p_{1}, p_{2}, \ldots, p_{n}\right)=-\sum_{k=1}^{n} p_{k} \log \left(p_{k}\right)
$$

The role of differential entropy as a measure of uncertainty in residual life time distribution has attracted increasing attention in recent years. As urged by Ebrahimi[7], if a unit is known to have survived to age $t$, then $H(X)$ is no longer useful for measuring the uncertainty about the remaining life time of the unit. Accordingly, he introduced the measure of uncertainty of residual life time distribution, $H(X ; t)$ of a component as, 


$$
H(X ; t)=-\int_{t}^{\infty} \frac{f(x)}{R(t)} \log \left(\frac{f(x)}{R(t)}\right) d x=1-\frac{1}{R(t)} \int_{t}^{\infty} \log r_{F}(x) d x
$$

where $R(t)$ is the survival function and $r_{F}(t)=\frac{f(t)}{R(t)}$ is the hazard function of the random variable $X$, because the reliability function $r_{F}(t)$ and the hazard rate function $h(t)$ can be uniquely determined from each other, a new ageing distribution can therefore be derived by constructing one of them first.

After the unit has survived for time $t, H(X ; t)$ basically measures the expected uncertainty in the conditional density of $(X / X>t)$ about the predictability of remaining life time of the unit .

It is reasonable to presume that in many realistic situations uncertainty is not necessarily related to the future but can also refer to the past. For instance, if at time $t$, a system which is observed only at certain preassigned inspection times, is found to be down, then the uncertainty of the system life relies on the past, i.e., on which instant in $(0, t)$ it has failed. Based on this idea, Dicrescenzo and Longobardi[5] have studied the past entropy over $(0, t)$. If $X$ denote the life time of a component or of living organism, then the past entropy of $X$ is defined as

$$
H^{o}(X ; t)=-\int_{0}^{t} \frac{f(x)}{F(t)} \log \left(\frac{f(x)}{F(t)}\right) d x=1-\frac{1}{F(t)} \int_{0}^{t} f(x) \log \tau(x) d x
$$

where $F(t)$ is the cumulative distribution function and $\tau(t)=\frac{f(t)}{F(t)}$ is the reversed hazard function or reversed failure rate of $X$.

The function $\tau(t)$ is receiving increasing attention in reliability theory and survival analysis $([4],[17])$. In view of the growing importance of the concept of reversed hazard function, Chandra and Roy [4] examined some result on implicative relationship in the context of the monotonic behavior of reversed hazard function.

In this paper, we investigate the problem of characterization of past life time distribution by using the following generalized residual entropy function,

$$
H_{\alpha}^{o}(X ; t)=\frac{1}{\alpha(1-\alpha)} \log \left(\frac{\int_{0}^{t} f(x) d x}{F(t)}\right)^{\alpha}, \alpha \neq 0,1 \text { and } \alpha>1
$$

It must to be noted that $\alpha \rightarrow 1$ and $t \rightarrow \infty \quad(1.5)$ reduce to (1.1). The measure (1.5) is the past residual life entropy corresponding to $(1.3)$.

\section{A Few Orders Based on the Generalised Past Entropy}

In this section, we define a few orders based on the generalized past entropy and study their properties.

Definition 2.1: Let $X$ and $Y$ be two random variables denoting the life time of two components with density functions $f$ and $g$ respectively. Then $X$ is said to be greater than $Y$ in past entropy (written as $\quad \stackrel{P E}{2} \geq$ ) if $H^{o}(X ; t) \leq H^{o}(Y ; t)$ for all $t \geq 0$

Definition 2.2: Let $X$ and $Y$ be two random variables denoting the life time of two components with density functions $f$ and $g$ respectively. Then $X$ is said to be greater than $Y$ in generalized past entropy of order $\alpha$ ( written as $X \stackrel{G P E}{\geq} Y)$ if, $H_{\alpha}^{o}(X ; t) \leq H_{\alpha}^{o}(Y ; t)$ for all $t \geq 0$.

The following lemma which gives the value of the function $H_{\alpha}^{o}(X ; t)$ under the linear transformation, will be used in proving the upcoming theorem of this section.

Lemma 2.1: For an absolutely continuous random variable $X$, define $Z=a X+b$, where $a>0, b \geq 0$ are constants.

Then, for $t>b$,

$$
H_{\alpha}^{o}(Z ; t)=\frac{\log a}{\alpha}+H_{\alpha}^{o}\left(X ; \frac{t-b}{a}\right)
$$

Proof: We have,

$H_{\alpha}^{o}(X ; t)=\frac{1}{\alpha(1-\alpha)} \log \left(\frac{\int_{0}^{t} f(x) d x}{F(t)}\right)^{\alpha}, \alpha \neq 0,1$ and $\alpha>1$

Also, $Z=a X+b, a>0, b \geq 0$

Therefore, for $t \geq b$ we have,

$H_{\alpha}^{o}(Z ; t)=\frac{\log a}{\alpha}+H_{\alpha}^{o}\left(X ; \frac{t-b}{a}\right)$, which proves the lemma.

The following theorem shows that generalized past entropy order defined above is closed under increasing linear transformation. 
Theorem 2.1: Let $X$ and $Y$ be two absolutely continuous random variables, define $Z_{1}=a_{1} X+b_{1}$ and $Z_{2}=a_{2} Y+b_{2}$, $a_{1}, a_{2}>0$ and $b_{1}, b_{2} \geq 0$. Let (I) $X \stackrel{G P E}{\geq} Y$ (II) $a_{1} \geq a_{2}$ (III) $b_{1} \geq b_{2}$. Then $Z_{1} \stackrel{G P E}{\geq} Z_{2}$, if $H_{\alpha}^{o}(X ; t)$ or $H_{\alpha}^{o}(Y ; t)$ is increasing in $t>b_{1}$.

Proof: Suppose $H_{\alpha}^{o}(X ; t)$ is increasing in $t$.

Now $X \stackrel{G P E}{\geq} Y$ implies,

$$
H_{\alpha}^{o}\left(X ; \frac{t-b_{2}}{a_{2}}\right) \leq H_{\alpha}^{o}\left(Y ; \frac{t-b_{2}}{a_{2}}\right)
$$

Further, since $\frac{t-b_{1}}{a_{1}} \leq \frac{t-b_{2}}{a_{2}}$, we have

$$
H_{\alpha}^{o}\left(X ; \frac{t-b_{1}}{a_{1}}\right) \leq H_{\alpha}^{o}\left(X ; \frac{t-b_{2}}{a_{2}}\right)
$$

From (2.1) and (2.2), we have

$$
H_{\alpha}^{o}\left(X ; \frac{t-b_{1}}{a_{1}}\right) \leq H_{\alpha}^{o}\left(Y ; \frac{t-b_{2}}{a_{2}}\right)
$$

$$
Z_{1} \stackrel{G P E}{\geq} Z_{2}
$$

The proof of part second is similar and hence omitted.

Corollary 2.1: Let $X$ and $Y$ be two absolutely continuous random variables such that $X \stackrel{G P E}{\geq} Y$. Define $Z_{1}=a X+b$ and $Z_{2}=a Y+b, a>0$ and $b \geq 0$ are constants. Then $Z_{1} \stackrel{G P E}{\geq} Z_{2}$, if $H_{\alpha}^{o}(X ; t)$ or $H_{\alpha}^{o}(Y ; t)$ is increasing in $t>b$.

Now we state a more stronger result. The proof is simple and hence omitted.

Theorem 2.2: Let $X$ and $Y$ be two absolutely continuous random variables .Define $Z_{1}=a X+b$ and $Z_{2}=a Y+b$, $a>0$ and $b \geq 0$ are constants. Then, $Z_{1} \stackrel{G P E}{\geq} Z_{2}$ if $X \stackrel{G P E}{\geq} Y$ for $t>b$.

\section{Characterization Results}

\subsection{Continuous Case}

Let $X$ be a continuous non negative random variable representing component failure time with failure distribution function $F(t)=P(X \leq t)$.

After simplification of (1.7), we have

Using (2.3) and applying lemma 2.1, we have

$$
\int_{0}^{t} f^{\alpha}(x) d x=F^{\alpha}(t)\left\{\exp \left(\alpha(1-\alpha) H_{\alpha}^{o}(X ; t)\right)\right\}, \alpha \neq 0,1 \text { and } \alpha>1
$$

We now show that $H_{\alpha}^{o}(X ; t)$ uniquely determines $F(t)$.

Theorem 3.1: Let $X$ be a continuous non negative random variable with distribution function $F(t)$ and an increasing generalized past residual entropy, $H_{\alpha}^{o}(X ; t)$. Then

$H_{\alpha}^{o}(X ; t)$ uniquely determines $F(t)$.

Proof: Differentiating (2.1) with respect to $t$, we have

$$
\tau^{\alpha}(t)=\alpha \tau(t)\left\{\exp \left(\alpha(1-\alpha) H_{\alpha}^{o}(X ; t)\right)\right\}+\alpha(1-\alpha) H_{\alpha}^{\prime o}(X ; t)\left\{\exp \left(\alpha(1-\alpha) H_{\alpha}^{o}(X ; t)\right)\right\}
$$

where $\tau(t)=\frac{f(t)}{F(t)}$ is the reversed hazard function.

Hence for fixed $t, \tau(t)$ is a solution of

$$
\psi(x)=x^{\alpha}-\alpha x\left\{\exp \left(\alpha(1-\alpha) H_{\alpha}^{o}(X ; t)\right)\right\}-\alpha(1-\alpha) H_{\alpha}^{\prime o}(X ; t)\left\{\exp \left(\alpha(1-\alpha) H_{\alpha}^{o}(X ; t)\right)\right\}=0
$$

Differentiating both sides with respect to $x$, we have

$$
\psi^{\prime}(x)=\alpha x^{\alpha-1}-\alpha\left\{\exp \left(\alpha(1-\alpha) H_{\alpha}^{o}(X ; t)\right)\right\}
$$

Note that $\psi^{\prime}(x)=0$ gives,

$$
x=\exp \left(-\alpha\left(H_{\alpha}^{o}(X ; t)\right)\right)=x_{t}
$$

Two cases arises:

Case 1: Let $\alpha>1$, then $\psi(0)>0$, and $\psi(x)$ is a convex function with minimum at

$x_{t}=\exp \left(-\alpha\left(H_{\alpha}^{o}(X ; t)\right)\right)$. So the unique solution to $\psi(x)=0$ is given by $x=\tau(t)$

Case 2: Let $\alpha<1$, then $\psi(0)<0$, and $\psi(x)$ is a concave function with maximum at

$x_{t}=\exp \left(-\alpha\left(H_{\alpha}^{o}(X ; t)\right)\right)$. So the unique solution to $\psi(x)=0$ is given by $x=\tau(t)$

Combining both the cases, we conclude that $H_{\alpha}^{o}(X ; t)$ uniquely determines $\tau(t)$, which in turns determines $F(t)$ uniquely.

Theorem 3.2: The uniform distribution over $(a, b), a<b$, can be characterized by increasing generalized past entropy 
$H_{\alpha}^{o}(X ; t)=\frac{1}{\alpha} \log (t-a), t>a$.

Proof: In case of uniform distribution over $(a, b), a<b$, we have

$H_{\alpha}^{o}(X ; t)=\frac{1}{\alpha} \log (t-a)$, which is increasing in $t>a$.

Differentiating with respect to $t$, we have $H_{\alpha}^{\prime o}(X ; t)=\frac{1}{\alpha(t-a)}$

Now, $x_{t}=\exp \left(-\alpha\left(H_{\alpha}^{o}(X ; t)\right)\right)=(t-a)^{-1}$
Using the value of $x_{t}$ in (3.2), we get $\psi\left(x_{t}\right)=0$

Thus, $\psi(x)=0$ has unique solution given by $x=x_{t}$. But $\tau(t)$ is a solution to (3.2). Hence, $\tau_{t}=x_{t}=(t-a)^{-1}, t>a$ is the unique solution to $\psi(x)=0$. This gives that the distribution is uniform, and the theorem is proved.

Belzunce et al. [3], derive the residual entropy expressions for some continuous distributions. Corresponding to these distributions, we derive the expressions for the generalized past residual entropy as mentioned in the below table A.

Table A. Generalised past residual entropy of various models

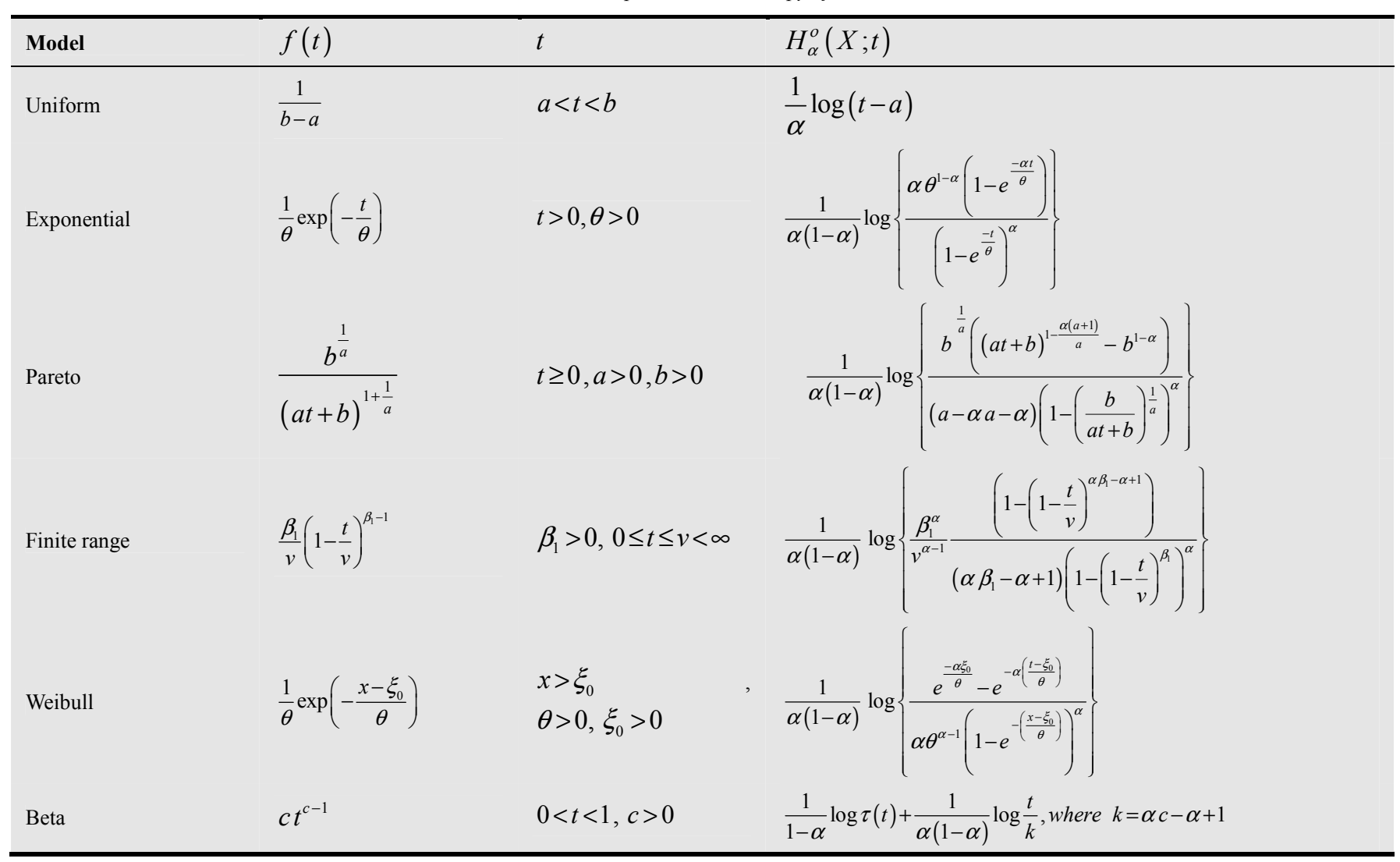

\subsection{Discrete Case}

Let $X$ is a discrete random variable taking the values $x_{0}, x_{1}, x_{2}, \ldots, x_{n} \quad$ with respective probabilities

$$
H_{\alpha}^{o}(p ; j)=-\sum_{k=0}^{j} \frac{p_{k}}{p(j)} \log \left(\frac{p_{k}}{p(j)}\right)
$$
$p_{0}, p_{1}, p_{2}, \ldots, p_{n}$. The past uncertainty of discrete lifetime distribution is defined as

where $p(j)=\sum_{k=0}^{j} p_{k}$ is the distribution function of $X$.

The generalized past residual entropy for discrete case is defined as

$$
H_{\alpha}^{o}(p ; j)=\frac{1}{\alpha(1-\alpha)} \log \left[\sum_{k=0}^{j}\left(\frac{p_{k}}{p(j)}\right)^{\alpha}\right], \alpha \neq 0,1 \text { and } \alpha>1
$$

for $\alpha \rightarrow 1$ (3.4) reduces to (3.3)

$F(t)$ with support $\{0,1,2, \ldots, n\}$ and an increasing

Theorem3.3: if $X$ has a discrete distribution function 
generalized past residual entropy $H_{\alpha}^{o}(p ; j)$, then $H_{\alpha}^{o}(p ; j)$ uniquely determines $F(t)$.

Proof : we have $H_{\alpha}^{o}(p ; j)=\frac{1}{\alpha(1-\alpha)} \log \left[\sum_{k=0}^{j}\left(\frac{p_{k}}{p(j)}\right)^{\alpha}\right]$, $\alpha \neq 0,1$ and $\alpha>1$

Which is equivalent to

$$
\sum_{k=0}^{j} p_{k}^{\alpha}=p^{\alpha}(j) \exp \left(\alpha(1-\alpha) H_{\alpha}^{o}(p ; j)\right)
$$

For $j+1$, we obtain

$$
\sum_{k=0}^{j+1} p_{k}^{\alpha}=p^{\alpha}(j+1) \exp \left(\alpha(1-\alpha) H_{\alpha}^{o}(p ; j+1)\right)
$$

Subtracting (3.5) from (3.6), writing $p_{j+1}=p(j+1)-p(j)$, we have

$$
\exp \left(\alpha(1-\alpha) H_{\alpha}^{o}(p ; j+1)\right)=\left(1-\theta_{j}\right)^{\alpha}+\theta_{j}^{\alpha} \exp \left(\alpha(1-\alpha) H_{\alpha}^{o}(p ; j)\right)
$$

where $\theta_{j}=\frac{p(j)}{p(j+1)} \in(0,1)$. It can be noted that for a fixed

$x>0, x=\theta_{j}$ is a solution to

$$
h(x)=(1-x)^{\alpha}+x^{\alpha} \exp \left(\alpha(1-\alpha) H_{\alpha}^{o}(p ; j)\right)-\exp \left(\alpha(1-\alpha) H_{\alpha}^{o}(p ; j+1)\right)=0
$$

Differentiating both sides with respect to $x$, we get $h^{\prime}(x)=-\alpha(1-x)^{\alpha-1}+\alpha x^{\alpha-1} \exp \left(\alpha(1-\alpha) H_{\alpha}^{o}(p ; j)\right)$

Thus $h^{\prime}(x)=0$, gives $x=\left[1+\exp \left(-\alpha H_{\alpha}^{o}(p ; j)\right)\right]^{-1}=x_{j}$

Two cases arises:

Case 1: When $\alpha>1, h(0)>0, h(1)>0$. Thus $h(x)$ first decreases then increases with minimum at $x_{j}=\left[1+\exp \left(-\alpha H_{\alpha}^{o}(p ; j)\right)\right]^{-1}$

Case 2: When $\alpha<1, h(0)<0, h(1)<0$. Thus $h(x)$ first increases and then decreases with maximum at $x_{j}=\left[1+\exp \left(-\alpha H_{\alpha}^{o}(p ; j)\right)\right]^{-1}$.

Combining both the cases, we conclude that $H_{\alpha}^{o}(p ; j)$ uniquely determines $\theta_{j}$, which in turns determines $F(t)$ uniquely.

Theorem 3.4: The uniform distribution with support $\{0,1,2, \ldots, n\}$ is characterized by increasing discrete generalized past residual entropy $H_{\alpha}^{o}(P ; j)=\frac{1}{\alpha} \log (j+1)$.

Proof: In case of uniform distribution with support $\{0,1,2, \ldots, n\}$, we have

$$
H_{\alpha}^{o}(P ; j)=\frac{1}{\alpha} \log (j+1) \quad, \quad j=0,1,2, \ldots, n \quad \text { which } \quad \text { is }
$$

increasing in $j$.

$$
\text { Also, } x_{j}=\left[1+\exp \left(-\alpha H_{\alpha}^{o}(p ; j)\right)\right]^{-1}=\left(\frac{j+1}{j+2}\right)
$$

therefore, $h\left(x_{j}\right)=0$

Thus $h(x)=0$ has a unique solution given by $x=x_{j}$. But $\theta_{j}$ is the solution of (3.8). Hence $\theta_{j}=\frac{p(j)}{p(j+1)}$ is the unique solution to $h(x)=0$. This gives that the distribution is discrete uniform and the theorem is proved.

\section{New Class of Life Time Distributions}

In this section we define a non parametric class of life time distributions based on generalized past residual entropy.

Definition: A non negative random variable $X$ is said to have increasing uncertainty of past life (IUPL), if $H^{o}(X ; t)$ is increasing in $t \geq 0$.

Definition: A non negative random variable $X$ is said to have increasing uncertainty of generalized past residual life (IUGPRL) of order $\alpha$, if $H_{\alpha}^{o}(X ; t)$ is increasing in $t \geq 0$.

Theorem 4.1: Let $X \in(I U G P R L)$ of order $\alpha$. Define $Z=a X+b$, where $a>0, b \geq 0$ are constants. Then $Z \in(I U G P R L)$.

Proof: Since $X \in(I U G P R L)$ of order $\alpha$,therefore, $H_{\alpha}^{\prime o}(X ; t) \geq 0$.

By applying lemma (2.1), it follows that $Z \in(I U G P R L)$ of order $\alpha$.

The next theorem gives the lower bound to the reversed hazard rate function in terms of $H_{\alpha}^{o}(X ; t)$.

Theorem 4.2 : If $X$ is IUGPRL of order $\alpha$, then $\tau(t) \geq \alpha^{\frac{1}{\alpha-1}} \exp \left(-\alpha H_{\alpha}^{o}(X ; t)\right)$.

Proof: Since $X$ is IUGPRL of order $\alpha$, therefore 
$H_{\alpha}^{\prime o}(X ; t) \geq 0$, which yields $\tau(t) \geq \alpha^{\frac{1}{\alpha-1}} \exp \left(-\alpha H_{\alpha}^{o}(X ; t)\right)$,

which proves the theorem.

Corollary 4.1: Let $F(t)$ be a is IUGPRL of order $\alpha$, then $F(t) \leq \exp \left\{-\int_{t}^{\infty}(\alpha)^{\frac{1}{\alpha-1}} e^{-\alpha H_{\alpha}^{o}(X ; u)} d u\right\}$

\section{Applications}

It is reasonable to presume that in many realistic situations uncertainty is not necessary related to the future but can also refer to the past. For instance, if at time, a system which is observed only at certain pre-assigned inspection times is found to be down, then the uncertainty of the system life relies on the past, i.e., on which instant in $(0, t)$ it has failed.

\subsection{Reliability}

Ageing distributions are an integral part of reliability engineering both at the component and system levels. Their applications include (but not limited to) lifetime analysis, accelerated life test, product burn-in before field use, replacement and warranty policies and many other issues.

\subsection{Actuarial Sciences}

The results of analysis are dependent upon the underlying assumptions involved, and the actuary should attempt to be as "unprejudiced" as possible in determining the modeling assumptions. In fact, this is actually a further strength of the information theoretic technique. The purpose is to investigate the effect of inflation, truncation or censoring from below (use of a deductible) and truncation or censoring from above (use of a policy limit) on the entropy of losses of insurance policies. Losses are differentiated between per-payment and per-loss (franchise deductible).

\subsection{Social Sciences}

In social sciences, the time spent (duration) in a state before exiting is an important consideration for managers and planners. For example, a social scientist may be concerned with the recidivism in criminal justice, the length of time to complete a $\mathrm{Ph}$. D degree, the duration an individual remains unemployed, the duration an individual stays in an employment, the duration an individual remains married, the durations of coalitions, the time until announcement of support of a bill, the length a leader stays in power, the duration of a war and others. Surprisingly, many such 'duration' variables have a unimodal hazard rate that can be fitted by the inverse-Weibull, inverse Gaussian, lognormal, exponential or generalized power Weibull family.

\subsection{Biological Systems}

Biological systems produce work through their lifetime. This generates Entropy expressed by the energy of decay of the system. Aging and the deterioration of the human body, diseases and organic decay are typical examples of various forms of Entropy. Medicine has being struggling to reduce their effect by optimizing the duration of human life.

\subsection{Ecosystems}

The energy produced by the work of living organisms generates Entropy in the form of decline of reproduction of species and the decay of their environment. This may be measured analytically, by the entropy which expresses the irreversible residue of low level energy. The phenomenon of such devastation is obvious in the environment which is full of waste dumps and barren land where various endangered species used to live.

\section{Conclusion}

We have introduced and studied the concept of entropy for the past life time distributions. In this context we study the properties of the resulting entropies such as the residual loss entropy and the past loss entropy which are the result of use of a deductible and a policy limit, respectively. Interesting relationships between these entropies are presented. We have generalized several results available in the literature regarding the past residual entropy. It is shown that the proposed measure uniquely determines the distribution function. The uniform and discrete uniform distribution has been characterized in terms of past-generalized residual entropy.

The theoretical results obtained in this paper can be used to go further and analyze the applications in other disciplines where uncertainty exists.

\section{Acknowledgements}

We sincerely thank the editor and the anonymous referees and reviewers, careful reading of the manuscript and some thought provoking comments. The valuable comments have forced us to give clarification to quite a few questions and to correct some mistakes improving the quality of the paper substantially.

\section{References}

[1] Asaid, M. and Ebrahimi, N., (2000), "Residual entropy and its characterizations in terms of hazard function and mean residual life function". Statist. Prob. Lett. 49: 263-269.

[2] Awad, A.M., (1987), "A statistical information measure". Dirasat XIV (12): 7- 20

[3] Belzunce, F., Navarror, J., Ruiz, J.M. and Aguila, Y., (2004), "Some results on residual entropy function".Metrika 59:147-161.

[4] Chandra, N.K. and Roy, D., (2001), "Some results on reversed hazard rate”. Prob. Eng. Inf. Sci. 15: 95-102.

[5] Crescenzo, A. D. and Longobardi, M., (2002), "Entropy based measure of uncertainty in past life time distributions". J.of applied probability 39 : 434-440. 
[6] Crescenzo, A.D. and Longobardi, M., (2004), "A measure of discrimination between past life time distributions" Stat. Prob.Lett. 67: 173-182

[7] Ebrahimi, N., (1996), "How to measure uncertainty in the life time distributions".Sankhya. vol. 58, Ser.A 48-57

[8] Ebrahimi, N., (1997) "Testing whether life time distribution is decreasing uncertainty”.J.Statist.Plann.Infer. 64:9-19

[9] Ebrahimi, N. and Kirmani, S.N.U.A., (1996), "Some results on ordering of survival function through uncertainty". Statist. Prob.Lett. 29:167-176

[10] Ebrahimi, N. and Pellerey, F., (1995), "New partial ordering of survival functions based on the notion of uncertainty". Journal of Applied probability. 32: 202-211

[11] Gupta, R.D. and Nanda, A.K., (2002), “ $\alpha$ - and $\beta-$ entropies and relative entropies ofdistributions".J.of Statistical Theory and Applications 3:177-190

[12] Hart, P.E., (1975), "Moment distributions in economics, an exposition". Journal of Royal Statistical Society, Series A 138: 423-434.

[13] Kapur, J.N., (1994), "Measures of information and their applications". Willy Eastern Limited.

[14] Kapur, J.N. and Kesavan, H.K., (1990), "Generalized maximum entropy principle withapplications". Sand-Ford Educational Press of the University of Waterloo, Canada.

[15] Kurths, J., Voss, A., Saparin, P., Witt, A., Klein, H.j., and Wessel, N., (1995), "QuantitativeAnalysis of heart rate variability". Chaos 1: 88-94

[16] Morales, D., Pardo, L., and Vajda, I., (1997), "Some new statistics for testing hypotheses inparametric models". Journal of Multivariate Analysis, 62: 137-168.

[17] Nair, K.R.M. and Rajesh, G., (1998), "Characterization of probability distributions using theresidual entropy function”.J.Indian Statist.Assoc. 36:157-166

[18] Nanda, A.K. and Paul, P., (2006) "Some properties of past entropy and their applactions".Metrika. 64: 47-61

[19] Nanda, A.K. and Paul, P., (2006) "Some results on generalized residual entropy".Information Science 176: 27-47.

[20] Nanda, A.K. and Paul, P., (2006) "Some results on generalized past entropy" .Journal of StatisticalPlanning and Inference. $136: 3659-3674$.

[21] Renyi, A., (1961), "On measure of entropy and information".Proceeding of the FourthBerkeley Symposium on Math. Statist. Prob.Vol 1, University of California Press, Berkely,547-561.

[22] Sankaran, P.G. and Gupta, R.P., (1999), "Characterization of the life time distributions using measure of uncertainty". Calcutta Statistical Association Bulletin. 49:154-166.

[23] Shannon, C.E.,(1948), "A mathematical theory of communication”. Bell System TechnicalJ. 27:379-423.
[24] Sankaran, P.G. and Gleeja, V.L., (2006), “On bivariate reversed hazard rates". J.Japan Statist. Soc. 36: 213-224.

[25] Song, K., (2001), "Renyi information, loglikelihood and an intrinsic distributionmeasure". Journal of Statistical Planning and inference 93: 51-69

[26] Jiang R, Murthy DNP. Impact of quality variations on product reliability. Reliability Engineering and System Safety 2009; 94:490-496.

[27] Wong KL. A new framework for part failure-rate prediction models. IEEE Transactions on Reliability 1995; 44(1):139-146.

[28] FinkelsteinM. Understanding the shape of the mixture failure rate (with engineering and demographic applications). Applied Stochastic Modelsin Business and Industry 2009; 25:643-663.

[29] Finkelstein M. Failure Rate Modeling for Reliability and Risk. Springer: London, 2008.

[30] Ghitany ME, Al-Awadhi FA, Alkhalfan LA. Marshall-Olkin extended Lomax distribution and its application. Communications in Statistics:Theory and Methods 2007; $36: 1855-1866$.

[31] Meeker WQ, Escobar LA. Statistical Methods for Reliability Data. John Wiley \& Sons: New York, 1998.

\section{Author's Biography}

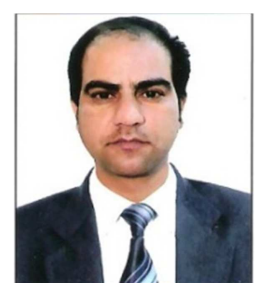

Dr. Arif Habib Hanga

Ph. D, M.Phil, M.Sc in Statistics; B. Sc., B. Ed., PGDCA in Computer Science.

I obtained my $\mathrm{Ph}$. D in statistics (Information theory), started my career as Chief Statistician, AYUSH, M. o. H, Government of India. In 2008, I joined as an Assistant professor in University of Kashmir. So far I have worked on 3 patents in my credit, published 14 papers, 4 monographs, and attended 28 National / International conferences. Presently I am working as Asstt. Professor in King Khalid University-Abha, KSA.

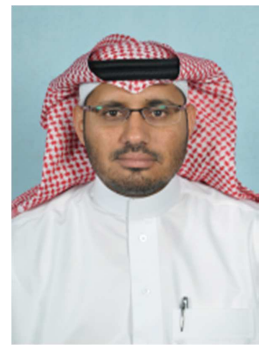

Mesheil Alahyani is Assistant Professor, College of Nursing, King Khalid University, KSA. He received his Bachelor of Nursing from Curtin University, Australia, an MSN (Edu) Curtin university and PhD (Quality of Care) from the same University. $\mathrm{He}$ is certified in Clinical Instructions and quality management. His expertise is in reliability and quality management and also has been recognized for his expertise in Hospitals Accreditations. 